\title{
Plataforma para la creación de audiojuegos: una solución mediante el uso de interfaces enactivas
}

\section{Platform for audiogames creation: an enactive interfaces}

\author{
based solution
}

Presentación: 06/10/2020

\section{Doctorando:}

\section{Guillermo Gilberto}

Centro de Investigación y Transferencia en Acústica (CINTRA), Universidad Tecnológica Nacional (UTN) - Consejo Nacional de Investigaciones Científicas y Técnicas de Argentina (CONICET) - Argentina

Igilberto@frc.utn.edu.ar

\section{Director:}

\section{Cristian D. García Bauza}

\section{Co-directores:}

\section{Fernando R. Bermejo - Fabián C. Tommasini}

\section{Resumen}

Se presentan en este trabajo los principales avances del segundo año del proyecto de tesis "Plataforma para la creación de audiojuegos: una solución mediante el uso de interfaces enactivas”, de la carrera Doctorado en Ingeniería mención Electrónica, de la Universidad Tecnológica Nacional, Facultad Regional Córdoba. Este proyecto busca contribuir al conocimiento en el diseño de tecnologías de apoyo e inclusión a personas con diversidad funcional visual. Se plantea la investigación y desarrollo de una solución integrada de hardware, software y diferentes aplicaciones (audiojuegos) guiada mediante el enfoque innovador provisto por teorías de cognición corporizada y de interfaces hombre máquina de tipo enactivas. Los objetivos planteados contemplan la generación de un espacio mediado tecnológicamente donde se habilite el entretenimiento, entrenamiento de habilidades de audición espacial e inclusión de personas con diferentes capacidades visuales.

Palabras clave: audiojuegos, interfaces enactivas, discapacidad visual, tecnologías asistivas.

\begin{abstract}
This paper presents the major advances achieved in the first phase of the thesis project named "Platform for audiogames creation: an enactive interfaces based solution", elaborated for the Electronics mention Engineering $\mathrm{PhD}$ of Universidad Tecnológica Nacional, Facultad Regional Córdoba. This projects aims to contribute on assistive and inclusion technologies knowledge, particularly to those that help visual impaired people. For this purpose investigation and development of an integrated hardware, software and different applications (audiogames) solution is proposed. Platform design is guided by novel embodied cognition theories and enactive type of human machine interfaces. Inclusive and technologically mediated playing area will be developed for entertainment, spatial audition abilities training and integration of sighted and visual impaired people.
\end{abstract}

Keywords: audiogames, enactive interfaces, visual impairment, assistive technologies. 


\section{Introducción}

Tradicionalmente se denomina Interfaces Humano-Máquina (IHM) al campo que se ocupa del diseño, implementación y evaluación de sistemas computacionales que son objeto de interacción con seres humanos (Hewett et al., 1992). Aunque el campo de estudio se ha visto enriquecido notablemente en las últimas décadas, la definición exacta de las IHM parece por momentos escurridiza, y el término muchas veces resulta un paraguas de uso común que abarca temas de estudio muy diversos. Ello provoca que se encuentren bajo este título diseños tecnológicos de muy variada complejidad, de muy diversas áreas de aplicación y de disímil éxito.

En el caso de las tecnologías asistivas a personas con discapacidad visual se observan grandes esfuerzos por desarrollar complejos dispositivos que ayuden a la movilidad y orientación independiente de la persona. Sin embargo, existe un consenso general en que la apropiación de tales dispositivos por parte de los usuarios es baja (Lloyd-Esenkaya et al., 2020). Si bien no existe un único motivo de este fenómeno, en los últimos años han aparecido críticas al modelo mental humano utilizado para el diseño de IHM que pueden ayudar a esclarecer esta situación. Recientemente, desde las Ciencias Cognitivas se introducen perspectivas alternativas que, al proponer una mirada distinta sobre modelos tradicionales de la cognición, impactan significativamente en el diseño tecnológico (Froese, 2016). Las denominadas Interfaces Enactivas operacionalizan conceptos traídos de estas teorías en la interacción humano máquina.

Mediante este Plan de Tesis se utiliza el marco de la ingeniería como sustrato para articular una propuesta integral e interdisciplinaria (figura 1) que avance en esta línea de diseño tecnológico, particularmente abordando el diseño y desarrollo de juegos computacionales sin claves visuales (audiojuegos). Estos dispositivos utilizan claves auditivas para generar accesibilidad a personas con discapacidad visual y revisten de excelentes cualidades para el desarrollo y entrenamiento de habilidades psicomotrices, cognitivas y sociales (Mairena, 2009). Se presentan sintéticamente en este trabajo avances teóricos y técnicos relacionados con el diseño de estas aplicaciones, así como también avances en el desarrollo formal de la carrera doctoral. Las actividades realizadas por el doctorando se realizan desde el Centro de Investigación y Transferencia en Acústica de (CINTRA, CONICET-UTN) de la Regional Córdoba en conjunto con el área Media.Lab del Instituto PLADEMA (UNCPBA) de la ciudad de Tandil, Bs. As.

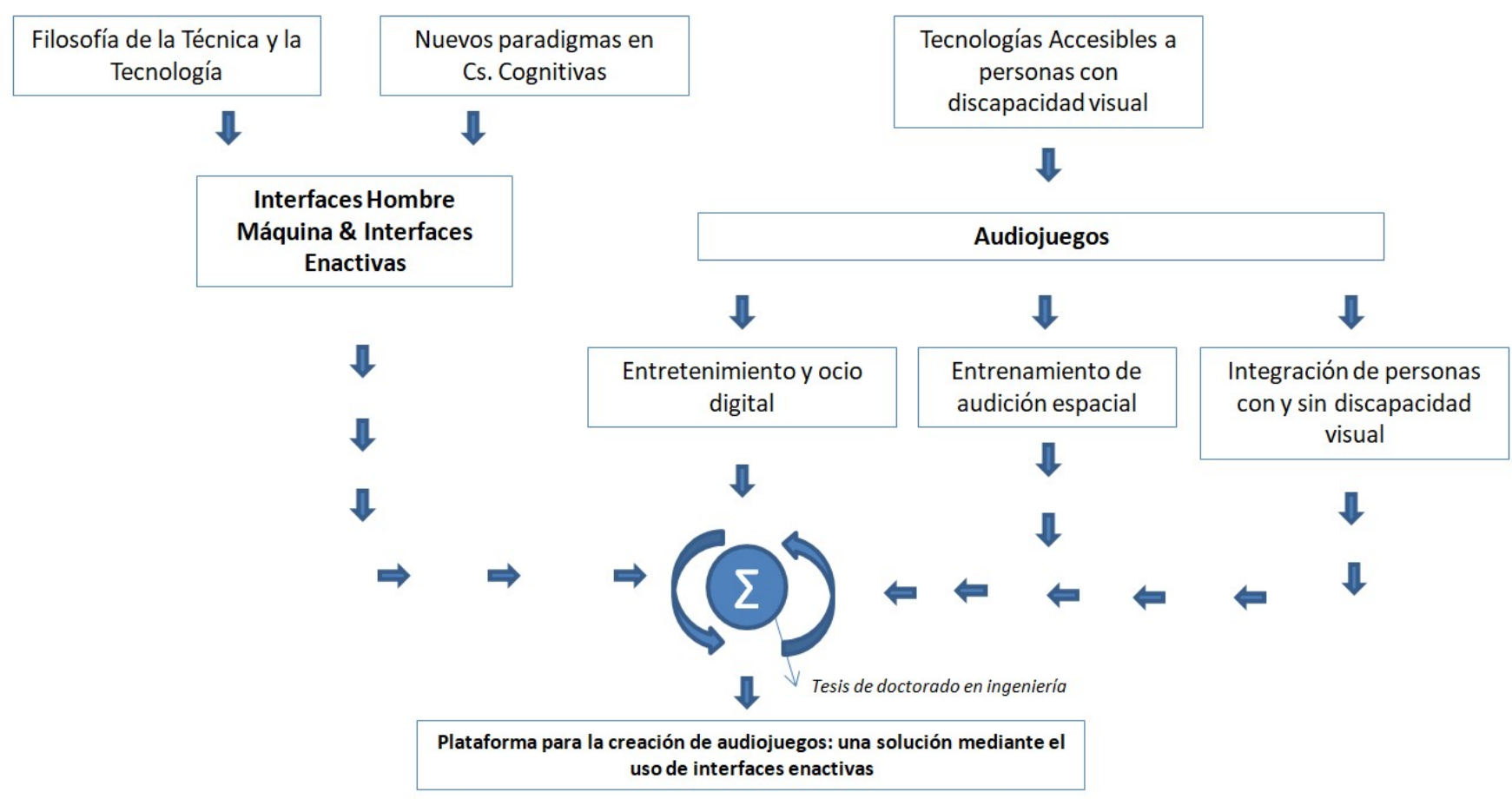

Figura 1: Propuesta interdisciplinaria para el diseño tecnológico de audiojuegos para personas con discapacidad visual. 


\section{Desarrollo}

\section{Modelos Cognitivos e Interfaces Hombre Máquina}

Se han identificado dos abordajes diferentes al diseño de interfaces tecnológicas devenidos de dos paradigmas de cognición humana diferentes (Froese, 2012). Por un lado, el enfoque más tradicional llamado "cognitivista", desde el cual se considera que la mente es una computadora que procesa símbolos abstractos. Los órganos sensoriales son periféricos encargados de recoger información del mundo exterior, mientras que el cerebro se encargaría de generar representaciones internas de ello para manipular con procesos lógico-matemáticos y elaborar una respuesta que se envía a los órganos efectores. Las IHM diseñadas con este enfoque apuntan a presentar al usuario datos abstractos, intentando maximizar el contenido y el flujo de información. El uso de estas interfaces requiere que el usuario aprenda a leer el código o lenguaje con el que trabaja. Suele decirse que son opacas, ya que requieren atención continua y reducen el contacto directo con el entorno. Ejemplos de estas interfaces relacionadas a la discapacidad visual pueden ser dispositivos como lectores de pantalla, procesadores de imágenes, navegadores satelitales, etc.

El segundo modelo está basado en nuevos enfoques de la cognición, especialmente en las perspectivas de la cognición extendida, situada, corporizada y enactiva (cognición 4e)(Newen et al., 2018). Sintéticamente se puede decir que estas perspectivas quitan el rol central del cerebro y entienden que hay una "extensión" de la cognición a los dispositivos técnicos utilizados por el hombre. Además, proponen que la cognición del agente es resultado de su corporeidad completa, su situación relacional con el entorno y de la capacidad sensoriomotora utilizada para conocer el entorno.

Las interfaces derivadas de estos modelos se denominan Interfaces Enactivas y privilegian la interacción directa, natural e intuitiva por parte del usuario (Khatchatourov, et al., 2007). La mediación tecnológica se da de manera tal que el ciclo sensoriomotor se ve intervenido a través de la incorporación transparente del dispositivo. Para lograr esta condición se evita cualquier carga reflexiva por parte del usuario para con la interfaz. El diseño de estos dispositivos debe promover que el usuario se "acople" con su entorno a través de una interacción activa, corporizada y continua, lo que implica la implementación de sistemas de lazo cerrado de rápida y permanente respuesta a las acciones del usuario. En ceguera, un dispositivo que cumple con estos criterios es el tradicional bastón blanco y algunos bastones aumentados mediante diferentes sensores e interfaces hápticas.

\section{Audiojuegos Enactivos}

Se están llevando adelante actividades para contribuir al diseño y desarrollo de audiojuegos accesibles basados en interfaces Enactivas que apunten al entretenimiento, entrenamiento de audición espacial e integración social. Para ello los trabajos se dividieron en dos líneas que se muestran a continuación:

Audiojuegos con auriculares estéreo: se diseñan entornos virtuales con sonido 3D para aplicaciones en teléfonos móviles con giróscopo. El celular se ubica solidariamente a la cabeza del jugador mediante una caja de realidad virtual. Los juegos utilizan la información de este sensor para actualizar en tiempo real la rotación de la cabeza del jugador dentro de la escena virtual. Mediante movimientos de cabeza el jugador puede obtener un registro auditivo panorámico de esta escena para realizar tareas que involucran localizar, reconocer y seguir fuentes sonoras. Se logra con esta configuración una interacción auditiva directa y natural, articulada con movimientos intuitivos propios de la escucha cotidiana. Las demás acciones llevadas adelante en el juego se basan en la manipulación de un joystick manual inalámbrico que cuenta con un pivot para trasladarse dentro de la escena y diferentes botones de acción.

Audiojuegos con sonido por altavoces distribuidos: Se diseñan entornos acústicos virtuales mediante altavoces en configuración estéreo y en cuadrafonía que se ejecutan desde una computadora. Estas disposiciones favorecen una interacción intuitiva y natural con el entorno sonoro, mejorando aún más la situación anterior ya que no se porta ningún dispositivo en los oídos. Inmersos en estos entornos, con libre movilidad en espacio relativamente amplio ( $2 \mathrm{~m}$ x $2 \mathrm{~m})$, los jugadores deben realizar diferentes tareas que implican localización y reconocimiento de fuentes sonoras. El sensado de movimiento mediante cámaras de profundidad de bajo costo (Kinect) permite tomar datos del jugador a distancia favoreciendo la transparencia del sistema, sin necesidad de costosos dispositivos. Los juegos que se están programando se basan en el acoplamiento entre las habilidades de 
audición espacial y movimientos corporales simples para señalar, cubrirse, mover objetos, etc. Se utiliza un joystick manual inalámbrico para sostener en una mano y ampliar las posibilidades de interacción.

En forma simultánea, en ambas líneas se están diseñando escenas de entrenamiento de complejidad creciente con el objetivo de disminuir progresivamente cualquier carga reflexiva puesta en la interfaz por parte del jugador. En las consignas de juego prevalece la estimulación a interactuar continuamente para favorecer la inmersión. Las tareas que el jugador debe realizar dentro de las escenas se relacionan mucho más con los movimientos que debe realizar, más que con procesos de decisión deductivos o inferenciales.

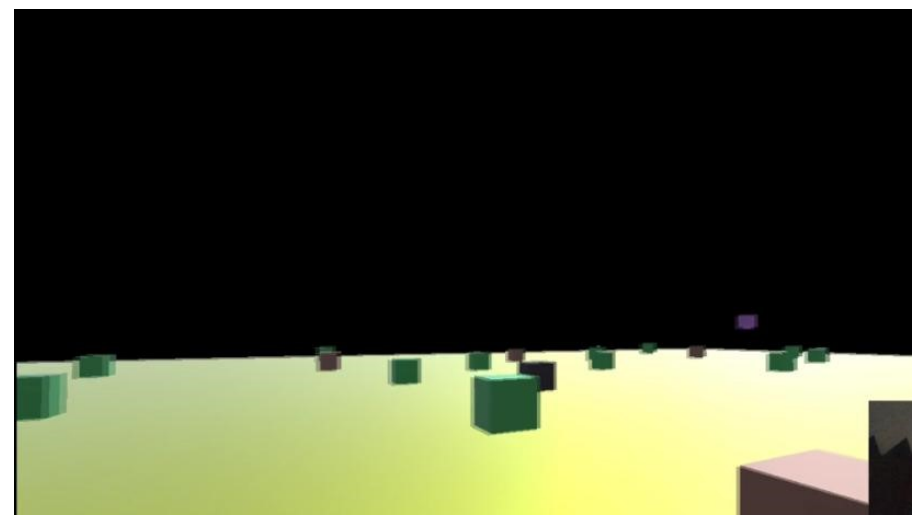

a)

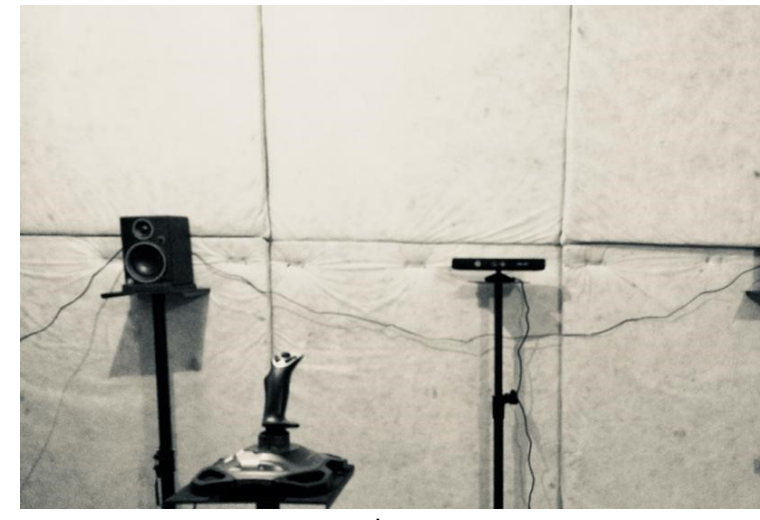

b)

Figura 2: a) Esquema visual del diseño de audiojuego para auriculares y teléfono celular solidario a movimientos de cabeza. Abajo a la izquierda se observa el jugador con la interfaz utilizada b) Interfaces utilizadas para los audiojuegos con altavoces distribuidos.

\section{Actividades complementarias de la formación doctoral}

- Presentación y aprobación del PID UTN 7667 “Aprendizaje Sensorio-Motor en Interacción: Plataforma de audiojuegos formativos para personas con y sin discapacidad visual": Este proyecto de investigación y desarrollo se desarrollo a partir de la primera etapa plan de trabajo doctoral. .Tiene una duración de dos años (2020-2021) y tiene como director al doctorando y como co-director al Dr. García Bauza (director del doctorando). El presupuesto asignado se utilzará para adquirir equipamiento de trabajo y costear reuniones y encuentros científicos.

- Dirección de becarios de grado: A partir de la homologación del PID mencionado se presentaron a la convocatoria de Becas de Rectorado dos postulantes para participar dentro del equipo de trabajo. Ambos fueron asignados con la beca. Cabe resaltar el caso de uno de ellos, estudiante ciego de Ingeniería en Sistema, que está trabajando activamente en la programación de las escenas de juego de las aplicaciones.

- Aprobación del curso de posgrado "Tecnologías Inmersivas para Proyectos Artísticos" , cursado a fines de 2019 y aprobado en febrero de 2020. El cursado se realizó presencial, viajando periódicamente a la Universidad Nacional de Quilmes, en el Gran Bs. As. En este cuso se adquirieron conocimientos fundamentales sobre diferentes herramientas para trabajar con realidad virtual. El trabajo final de este curso devino luego en una de las áreas de trabajo actuales presentada anteriormente (Audiojuegos con auriculares estéreo, para dispositivos móviles)

- Cursado del curso de posgrado "Filosofía de la Técnica y del Cambio Tecnológico" . Finalizado el cursado virtual, pendiente el trabajo final. Este curso pertenece a la Maestría en Tecnologías, Políticas y Cultura del Centro de Estudios Avanzados de la Universidad Nacional de Córdoba (UNC), en conjunto con sus Facultades de Ciencias Sociales y de Arte. Los docentes a cargo son referentes nacionales en el campo de la Filosofía de la Técnica (Lawler, Osella, Sandrone, Parente, D'Andrea). Se adquirieron conocimiento de categorías fundamentales en el estudio de la tecnología como fenómeno humano, la adquisición de herramientas críticas para evaluación y desarrollo de proyectos tecnológicos, la 
interacción con profesionales interesados en tecnología de una amplia gama de disciplinas y de distintos puntos del país, además de un gran enriquecimiento para el apoyo bibliográfico del proyecto.

- Envío de resumen extendido a las "Jornadas de Ciencia y Tecnología 2020" de la UTN, Facultad Regional San Francisco. En estas jornadas se realizará una presentación oral que condensa posturas de diferentes autores en referencia a IHM tradicionales y presentando el modelo de Interfaces Enactivas y el trabajo que se está realizando en torno al desarrollo de audiojuegos.

- Docente invitado de Práctica Profesional de Investigación de dos estudiantes de la Licenciatura en Psicología, de la Universidad Nacional de Córdoba. Este trabajo se realiza con el Dr. Bermejo (co-director del doctorando).

- Trabajos con estudiante avanzada de la carrera Ingeniería Biomédica de la UNC en la presentación de su anteproyecto final de carrera. Con esta estudiante se planea trabajar en colaboración para avanzar en la línea de audiojuegos con altavoces distribuidos, presentando como trabajo final el diseño, desarrollo y validación de una aplicación para el entrenamiento de audición espacial con Interfaces Enactivas

\section{Conclusiones}

La cognición 4E provee un novedoso marco para entender el diseño y uso de la tecnología. El objetivo ya no es diseñar IHM con las que se debe interactuar, sino más bien interfaces transparentes que sirven para generar nuevas formas de interactuar con el entorno. Entendemos que el diseño de audiojuegos puede beneficiarse de estas propuestas. Estos dispositivos, si bien poseen un gran potencial para acompañar el desarrollo de personas con discapacidad visual, precisan ofrecer una interactividad hombre-máquina superior. El enfoque de las Interfaces Enactivas ofrece interesantes criterios como la búsqueda por la naturalidad de la interacción, la prevalecencia de modos directos e intuitivos de percepción, la transparencia de los objetos técnicos y la búsqueda de un usuario activo interactuando de forma continua y corporizada.

Por su parte, tanto los avances teóricos y técnicos presentados como los referidos a las actividades formales de la carrera doctoral, dan cuenta de una adecuada posición del doctorado en relación con el $2^{\circ}$ año de la carrera doctoral. Si bien resta avanzar en profundidad en las áreas presentadas, los logros actuales son muy promisorios en relación con la concreción de los objetivos previstos.

\section{Referencias}

Hewett, T. T., Baecker, R., Card, S., Carey, T., Gasen, J., Mantei, M., ... \& Verplank, W. (1992). ACM SIGCHI curricula for human-computer interaction. ACM.

Lloyd-Esenkaya, T., et al. (2020). Multisensory inclusive design with sensory substitution. Cognitive Research: Principles and Implications, 5, 1-15.

Froese T., et al. (2012) The enactive torch: a new tool for the science of perception. IEEE Transactions on Haptics. 5, 365-375.

Froese, T. (2016) "De la cibernética a la nueva ciencia cognitiva." Revista Ciencia, 67, 51-58.

Mairena, J. (2009) "Videojuegos accesibles: por qué y cómo hacerlos" IV Congreso de la CiberSociedad.

Newen A., et. al., eds. (2018) The Oxford handbook of 4E cognition. Oxford University Press.

Khatchatourov, A., et al. (2007) Towards an enactive epistemology of technics. In Proceedings of ENACTIVE/07, 129-132. 